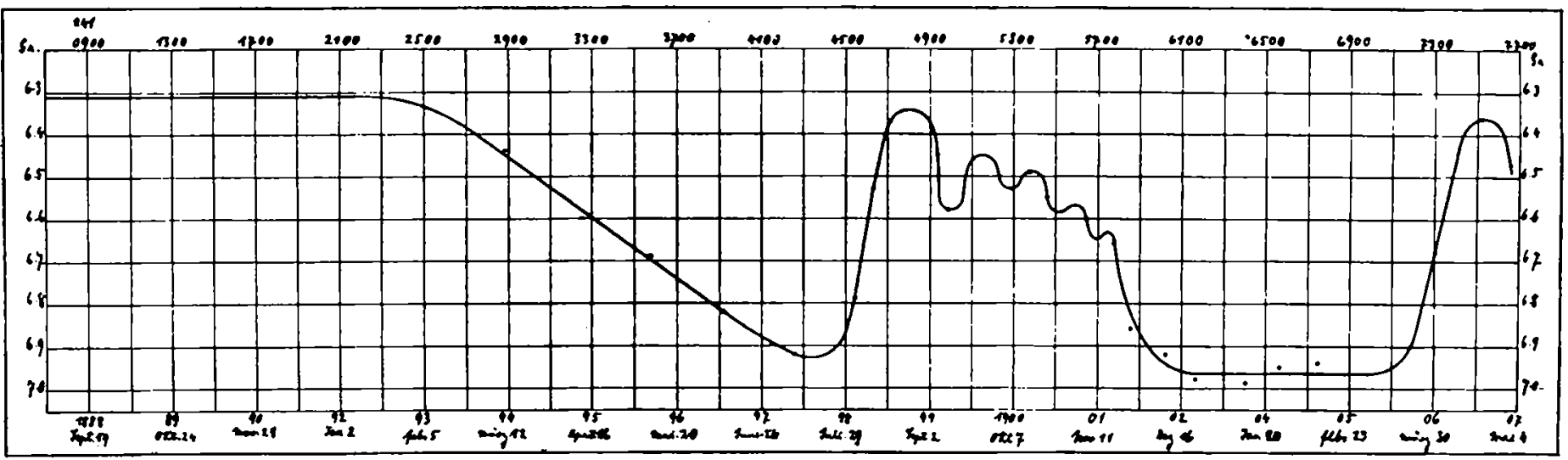

Die Helligkeiten des Veränderlichen ergeben sich vach / lesen aus der im Original in größerem Maßstabe gezeichder Kurve für die einzelnen Maxima und Minima etwas ver- neten Kurve ergeben.

schieden. Indessen betragen diese Unterschiede nur wenige Hundertstel einer Größenklasse und durfen daher nicht als verburgt angesehen werden, auch wurden sich die Differenzen leicht durch eine etwas andere Fuhrung der Kurve ausgleichen lassen.

Zum Schlusse mögen noch die Daten fưr die einzelnen Epochen zusammengestellt werden, wie sie sich beim Ab.

J. D.
2410693
2200
4340
4800
6160
7000
7520

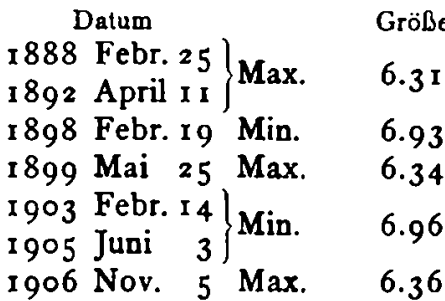

G. Miller und $P$. Kempf.

Potsdam, Astrophys. Observatorium, r 907 April 22.

\title{
Maxima et minima d'étoiles variables à longue période
}

par M. M. Luizet, à l'Observatoire de Lyon.

Dans le tableau ci-dessous je donne les dates des maxima ou des minima d'éclat de quelques étoiles variables à longue période, dates qui ont été tirées graphiquement de mes observations.

Les observations ont été faites par la méthode d'Argelander: à l'œil nu pour o Ceti, à l'aide d'une jumelle pour les maxima de $\mathrm{S}$ Coronae et de $\mathbf{R}$ Leonis, et a l'équatorial coude (ouverture $0.32 \mathrm{~m}$ ) pour tous les minima.

Les éclats de o Ceti ont été rapportés à ceux des étoiles suivantes:

\begin{tabular}{|c|c|c|c|c|c|}
\hline Nom. & Phase & Dates & Gr. & $O-C$ & Obs. \\
\hline o Ceti & Max. & 1897 Nov. 18 & $3^{m} \cdot 4$ & $+9^{j}$ & 15 \\
\hline , & $\bullet$ & I 898 Oct. I I & 2.9 & +5 & 37 \\
\hline , & , & r 899 Sept. 22 & 4.4 & +19 & $3^{2}$ \\
\hline • & 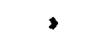 & I905 Mars 3 & 3.9 & +6 & I 3 \\
\hline • & 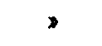 & I 906 Janv. I 5 & 4.8 & -7 & I I \\
\hline$\searrow$ & 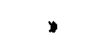 & , Déc. 18 & 2.3 & -2 & I 8 \\
\hline R Leonis & Max. & I905 Mai 14 & 5.8 & $-4 I$ & 20 \\
\hline , & . & I906 Avril I & 5.6 & $-3^{2}$ & 20 \\
\hline S Coronae & Max. & I902 Mars 25 & 7.2 & +9 & I 4 \\
\hline , & , & I903 Mars I 6 & $7 \cdot 1$ & +5 & 23 \\
\hline$>$ & 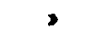 & 1904 Mars 12 & 6.4 & +6 & I 9 \\
\hline
\end{tabular}

\begin{tabular}{ll|ll}
$\gamma$ Orionis & $2 \mathrm{~m} .0$ & $\gamma$ Ceti & 3.8 \\
$\alpha$ Arietis & 2.2 & $\alpha$ Piscium & 4.1 \\
$\beta$ Ceti & 2.4 & $\delta$ Ceti & 4.6 \\
$\alpha$ Ceti & 2.9 & $\xi$ Piscium & 4.9
\end{tabular}

Les grandeurs maxima de $\mathrm{R}$ I,eonis et de $\mathrm{S}$ Coronae ont été obtenues en adoptant, pour les étoiles de comparaison, les grandeurs du catalogue photométrique de Potsdam; enfin, les éclats minima sont tous rapportés aux grandeurs adoptées par le P. Hagen dans son Atlas stellarum variabilium, ainsi que le maximum de $\mathrm{Z}$ Cygni.

\begin{tabular}{|c|c|c|c|c|c|}
\hline Nom. & Phase & Dates & Gr. & $\mathrm{O}-\mathrm{C}$ & Obs. \\
\hline S Coronae & Max. & 1905 Mars ro & $7: 6$ & $+10^{j}$ & 16 \\
\hline , & & I 906 Mars 30: & $7 \cdot 7$ & +35 & 12 \\
\hline , & Min. & 1906 Oct. 25 & 12.0 & +6 & $10 ́$ \\
\hline W Herculis & Min. & - Oct. 20 & I 2.1 & - 18 & 10 \\
\hline S Herculis & Min. & Oct. 4 & 12.0 & -20 & I I \\
\hline T Herculis & Min. & Nov. 2 & I I.8 & +7 & Io \\
\hline $\mathrm{R}$ Aquilae & Min. & Aout 25: & 10.2 & -28 & I 2 \\
\hline Z Cygni & Max. & , Sept. 3 & 8.9 & --40 & 15 \\
\hline R Delphini & Min. & - Juill. I & 13.0 & -22 & Io \\
\hline S Delphini & Min. & Dct. 6 & 11.1 & +2 & 12 \\
\hline S Lacertae & Min. & - Oct. 22 & 11.6 & +16 & 16 \\
\hline
\end{tabular}

Les différences $\mathrm{O}-\mathrm{C}$ ont été obtenues avec les éléments donnés dans le $3^{\ominus}$ catalogue de $\mathrm{M}$. Chandler.

St. Genis-Laval, I 907 Mars 15.

M. Luizet. 